\title{
The Response of the ITCZ to Extratropical Thermal Forcing: Idealized Slab-Ocean Experiments with a GCM
}

\author{
SARAH M. KANG \\ Program in Atmospheric and Oceanic Sciences, Princeton University, Princeton, New Jersey \\ ISAAC M. HELD \\ NOAA/Geophysical Fluid Dynamical Laboratory, Princeton, New Jersey \\ DARgAN M. W. Frierson \\ Department of Atmospheric Sciences, University of Washington, Seattle, Washington \\ MiNG ZHAO \\ Program in Atmospheric and Oceanic Sciences, Princeton University, Princeton, New Jersey
}

(Manuscript received 17 July 2007, in final form 7 December 2007)

\begin{abstract}
Using a comprehensive atmospheric GCM coupled to a slab mixed layer ocean, experiments are performed to study the mechanism by which displacements of the intertropical convergence zone (ITCZ) are forced from the extratropics. The northern extratropics are cooled and the southern extratropics are warmed by an imposed cross-equatorial flux beneath the mixed layer, forcing a southward shift in the ITCZ. The ITCZ displacement can be understood in terms of the degree of compensation between the imposed oceanic flux and the resulting response in the atmospheric energy transport in the tropics. The magnitude of the ITCZ displacement is very sensitive to a parameter in the convection scheme that limits the entrainment into convective plumes. The change in the convection scheme affects the extratropical-tropical interactions in the model primarily by modifying the cloud response. The results raise the possibility that the response of tropical precipitation to extratropical thermal forcing, important for a variety of problems in climate dynamics (such as the response of the tropics to the Northern Hemisphere ice sheets during glacial maxima or to variations in the Atlantic meridional overturning circulation), may be strongly dependent on cloud feedback. The model configuration described here is suggested as a useful benchmark helping to quantify extratropical-tropical interactions in atmospheric models.
\end{abstract}

\section{Introduction}

It is often assumed that the position of the ITCZ in the tropics is controlled by tropical mechanisms (Xie 2004). However, there is paleoclimatic and modeling evidence that one can alter the position of the ITCZ by perturbing the thermal forcing in the extratropics, with the ITCZ moving away from a cooled hemisphere or toward a warmed hemisphere. For example, Koutavas and Lynch-Stieglitz (2004) find that the marine ITCZ in the eastern Pacific is displaced southward when the

Corresponding author address: Sarah Kang, Princeton University, Forrestal Campus, 201 Forrestal Road, Princeton, NJ 08540. E-mail: skang@princeton.edu
Northern Hemisphere is cooled by ice sheets at glacial maxima. Evidence from the Cariaco Basin in the tropical Atlantic also indicates very strong coupling between tropical circulation and high-latitude climate change through the last glacial-interglacial transition (Lea et al. 2003). Modeling studies support the premise that the ITCZ is sensitive to ice cover (Chiang and Bitz 2005) and to the Atlantic thermohaline circulation (Zhang and Delworth 2005). In simulations with an atmospheric model coupled to a slab ocean, Broccoli et al. (2006) show that imposing antisymmetric interhemispheric heating in high latitudes induces a displacement of the ITCZ toward the warmer hemisphere.

The experimental configuration that we study here is similar to that of Broccoli et al. (2006) but is further 
idealized to help isolate factors controlling the strength of the tropical-extratropical interaction. An important feature of this model configuration is the use of a slabocean model as the lower boundary condition, so that the surface energy budget is closed.

\section{The basic GCM experiments}

The model employed in this study is AM2, an atmospheric general circulation model developed at the Geophysical Fluid Dynamics Laboratory (GFDL). The version utilized here is identical to that presented by Anderson et al. (2004). It has 24 vertical levels, with a horizontal resolution of $2^{\circ}$ latitude $\times 2.5^{\circ}$ longitude. The convective closure in the model is a modified version of the relaxed Arakawa-Schubert scheme of Moorthi and Suarez (1992). In this parameterization, convection is represented by a spectrum of entraining plumes. There is a plume corresponding to each model level above the cloud base for which there is sufficient buoyancy in the environment that it can be reached by an entraining plume. The entrainment rates in these plumes are determined by the requirement that the levels of neutral buoyancy correspond to model levels. Deep convection is prevented from occurring in updrafts with a lateral entrainment rate lower than a critical value $\lambda_{0}$, which is determined by the depth of the subcloud layer $z_{M}\left(\lambda_{0}=\alpha / z_{M}\right.$; Tokioka et al. 1988). As in Held et al. (2007), we find that $\alpha$ is an important parameter in this model; in particular, it controls the fraction of tropical precipitation that occurs through the large-scale cloud/condensation module as opposed to the convection module.

We consider aquaplanet simulations in which the atmosphere is coupled to a motionless slab ocean with a small heat capacity of $1 \times 10^{7} \mathrm{~J} \mathrm{~m}^{-2} \mathrm{~K}^{-1}$, corresponding to $2.4 \mathrm{~m}$ of water. This small heat capacity is chosen to reduce the time required for the model to reach equilibrium. To show the robustness of the results to the value of the mixed layer depth, we briefly show results from a 50-m depth simulation in section 3. In particular, the obliquity of the earth is set to zero, so there is no seasonal cycle; however, a diurnal cycle is retained, as in Neale and Hoskins (2000), but we also use a slab ocean rather than fixed surface temperatures, following the mixed layer benchmark proposed by Lee et al. (2008). Ocean temperatures are permitted to drop below freezing, and no sea ice is allowed to form. The simulations are run for $7 \mathrm{yr}$, and the last $4 \mathrm{yr}$ are used for averaging. Inspection of the energy balance and gross circulation features suggests that $3 \mathrm{yr}$ of spinup is more than adequate for reaching an equilibrium state in this configuration and that $4 \mathrm{yr}$ of averaging defines a statistically steady state with sufficient precision for our purposes.
The experiments are designed to study the mechanism by which ITCZ displacements are forced when heat is subtracted from one hemisphere and simultaneously added to the other hemisphere (equivalent to an imposed cross-equatorial heat flux in the ocean). Heating is imposed poleward of $40^{\circ} \mathrm{S}$, with equal and opposite cooling added poleward of $40^{\circ} \mathrm{N}$. The form of the heating is

$$
\begin{array}{ll}
H=-A \sin \left(\frac{\theta+40}{50} \pi\right) & \text { for }-90<\theta<-40, \\
H=-A \sin \left(\frac{\theta-40}{50} \pi\right) & \text { for } 40<\theta<90, \text { and } \\
H=0 & \text { otherwise, }
\end{array}
$$

where $A$ is the strength of the forcing ( $\mathrm{W} \mathrm{m}^{-2}$ ). The imposed forcing neither adds nor subtracts heat from the global system. Thus, the forcing can be described completely by an implied ocean flux $F_{0}$ from one hemisphere to the other, with $H=-\boldsymbol{\nabla} \cdot F_{0}$. Distributions of the heating/cooling $(H)$ and associated heat flux $\left(F_{0}\right)$ are plotted in Fig. 1.

We plot the tropical precipitation distribution with latitude for different values of $A$ in Fig. 2a and the corresponding distribution of SST with latitude in Fig. 2b. For the largest value of the forcing imposed $(A=$ 90), there is over a $30-\mathrm{K}$ change in SST in high latitudes. The ITCZ is shifted toward the warmer hemisphere (the Southern Hemisphere in these experiments) in all cases. With larger amplitude of external forcing, the shift of the ITCZ to the south grows monotonically, as does the changes in SST. While the ITCZ is shifted farther, the maximum precipitation gets weaker as the ITCZ widens somewhat. We would eventually like to understand these tropical responses to extratropical forcing quantitatively and to determine the extent to which they are model dependent.

In response to the extratropical forcing, the boundary between the Hadley cells moves south of the equator, the Hadley circulation in the cold Northern Hemisphere strengthens, and the cell in the warm Southern Hemisphere weakens. This shift of the boundary between two Hadley cells suggests that there are fluxes of energy from the south to the north to compensate for the imposed north-to-south oceanic flux. To help in understanding changes in the tropical circulation, we find it useful to measure the degree of compensation $C$ between the imposed oceanic flux and the resulting response in the atmospheric energy transport. The steady-state energy budget for the atmosphere over a mixed layer, denoting the vertical integral and zonal and time average with a bracket, is

$$
R_{\mathrm{TOA}}-\nabla \cdot F_{0}=\boldsymbol{\nabla} \cdot[m v],
$$


(a)

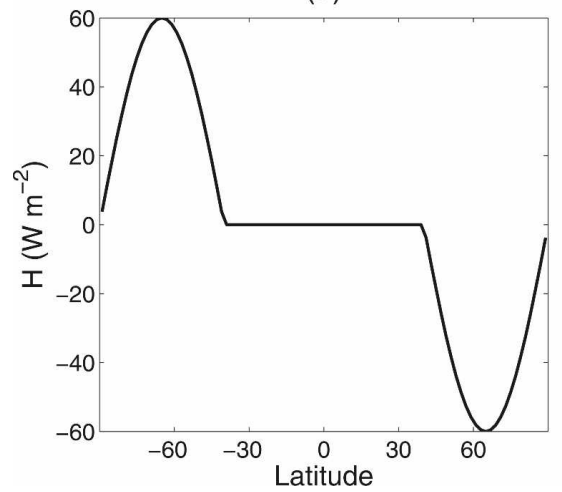

(b)

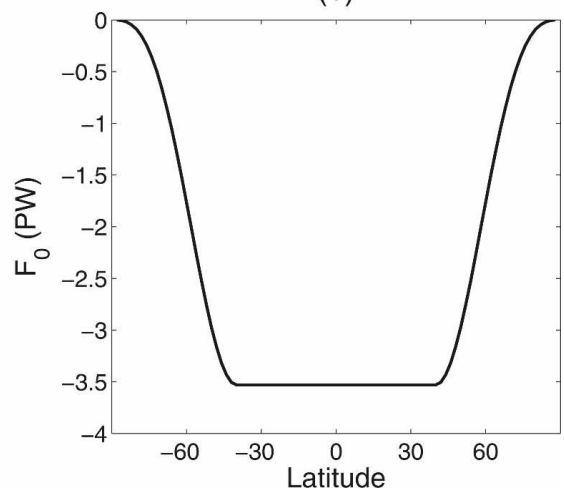

FIG. 1. (a) Latitudinal distribution of imposed forcing $H\left(\mathrm{~W} \mathrm{~m}^{-2}\right)$ and (b) associated implied ocean flux $F_{o}(\mathrm{PW})$ when $A=60 \mathrm{~W} \mathrm{~m}^{-2}$.

where $R_{\mathrm{TOA}}$ is the zonal and time mean incoming net radiation at top of the atmosphere (TOA), $F_{0}$ is the imposed cross-equatorial heat flux in the ocean, $m\left(=C_{p} T+\right.$ $\Phi+L q)$ is the moist static energy, and $v$ the meridional velocity. In the definition of moist static energy, $\Phi$ is the geopotential height, $L$ the latent heat of condensation, and $q$ the specific humidity. We set $F=[m v]$ and $C=\left|\left(F-F_{\text {ctl }}\right) / F_{0}\right|$, where the subscript ctl denotes the control case. Because the global mean of $R_{\mathrm{TOA}}$ is zero in a steady state, it can be represented in a flux form $F_{\mathrm{TOA}}$ (i.e., $R_{\mathrm{TOA}}=-\nabla \cdot F_{\mathrm{TOA}}$, the convergence of the total energy transport, both atmospheric and oceanic).

Equation (1) states that the imposed oceanic flux $F_{0}$ is balanced by changes in both the atmospheric energy transport $F$ and radiation $F_{\text {TOA }}$. The latter can be strongly influenced by clouds, which will be discussed in section 4. One can imagine an extreme case in which the response to the heating does not penetrate into the tropics and all of the imposed heating is balanced within the extratropics by TOA radiation. In this case the compensation $C$ is zero in the tropics. In fact, some of the response does penetrate into the tropics and there are changes in atmospheric meridional energy fluxes that compensate for part of the imposed oceanic flux, so that $C>0$. In regions where $C$ approaches unity, the imposed oceanic flux is closely compensated by the atmospheric energy fluxes. Figure 3 a compares $F-F_{\text {ctl }}$ (solid line), for the case $A=60$ and the control value of the convection parameter $\alpha$, with the imposed oceanic flux $F_{0}$ (dashed line). The degree of compensation varies somewhat with latitude near the equator, with a value of $87 \%$ when averaged from $20^{\circ} \mathrm{S}$ to $20^{\circ} \mathrm{N}$ in this case. We will return later to the other lines in Fig. 3.

We also define the latitude where the moist static energy flux $F$ is zero to be the "energy flux equator" $\theta_{F}$, (a)

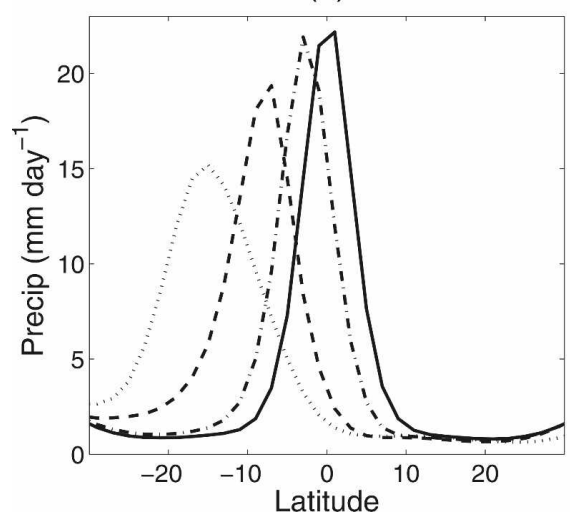

(b)

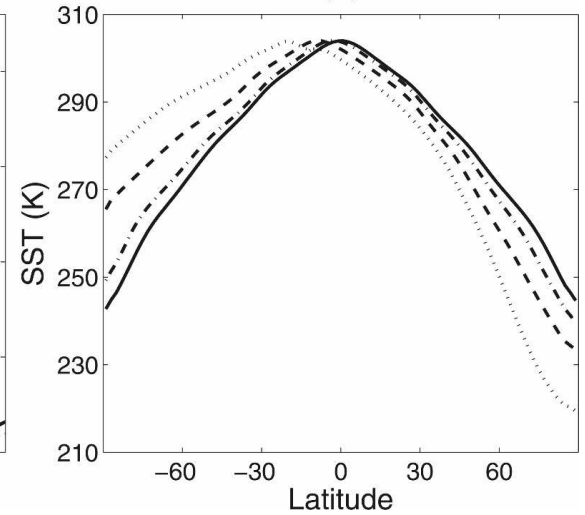

FIG. 2. Time mean, zonal mean (a) precipitation $\left(\mathrm{mm} \mathrm{day}^{-1}\right)$ in the tropics, and (b) SST (K) for $A=0$ (solid), 10 (dashed-dotted), 30 (dashed), and $60 \mathrm{~W} \mathrm{~m}^{-2}$ (dotted), with a control value of $\alpha(=1 X)$. 
(a)

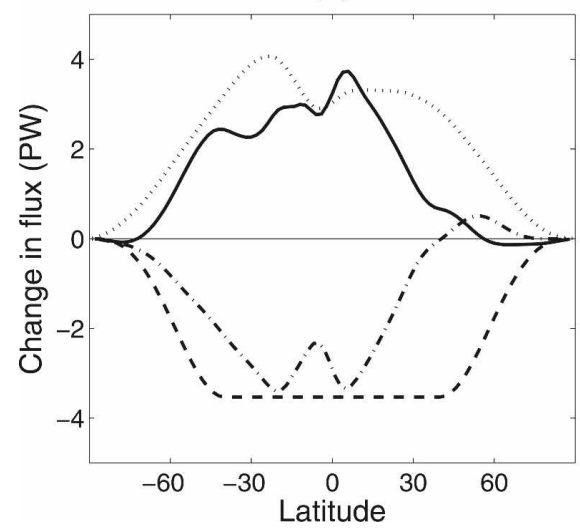

(b)

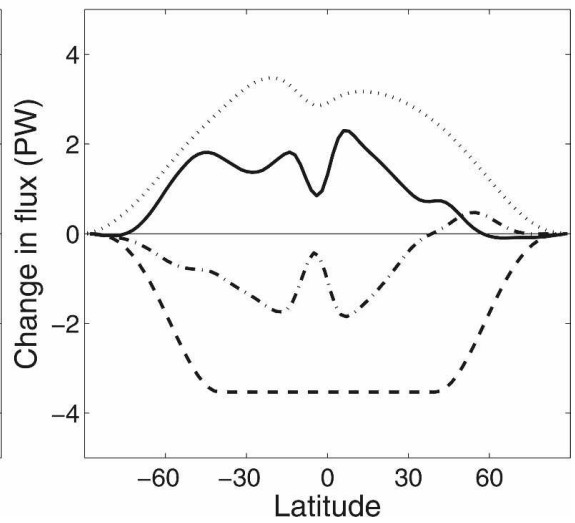

FIG. 3. The anomalous vertically integrated moist static energy transports, $F-F_{\mathrm{ctl}}(\mathrm{PW})$ for $A=60 \mathrm{~W} \mathrm{~m}^{-2}$ (solid), the imposed oceanic flux $F_{0}$ (dashed), the flux corresponding to the change in cloud radiative forcing $\delta F_{\mathrm{CRF}}$ (dashed-dotted) and clear-sky radiation $\delta F_{\text {clr }}$ (dotted), for (a) the control value of $\alpha(=1 X)$ and (b) $\alpha=10 X$.

which provides another way of quantifying the asymmetry in the atmospheric energy transport. The eddy energy fluxes in the tropics are small, so the energy flux equator coincides rather well with the boundary between the Hadley cells, and $C$ and $\theta_{F}$ are very closely related. From the definition of $C$, one can compute the energy flux equator by setting $F=F_{\text {ctl }}-C F_{0}$ and locating the zero in this flux. When a computation uses a value for $C$ averaged over some latitude band in the tropics, this expression is only approximate, but should still be useful. The larger the compensation by the atmospheric energy fluxes, the farther poleward the energy flux equator will be located.

Figure 4a shows the latitude of the ITCZ and the latitude of the energy flux equator as a function of the strength of the forcing $A$. The ITCZ location is defined as the location of the tropical maximum in precipitation, obtained by differentiating the precipitation with respect to latitude and linearly interpolating to obtain the zero crossing. The zero crossing of the atmospheric flux at the energy flux equator is obtained by linear interpolation as well. There are some differences between the ITCZ location and the energy flux equator, with the former moving farther from the equator than the latter as $A$ increases. In these experiments this difference is relatively small, and $\theta_{F}$ provides a useful estimate of the location of the ITCZ. This is not always the case in other models. For example, when the simplified moist GCM with gray radiative transfer as described by Frierson et al. (2006) is perturbed in the (a)

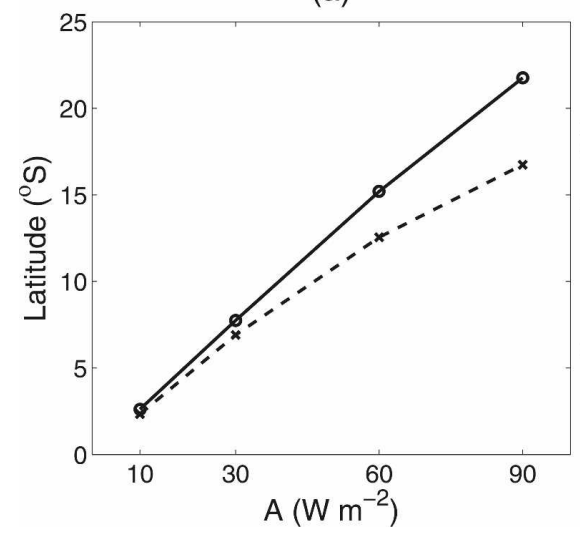

(b)

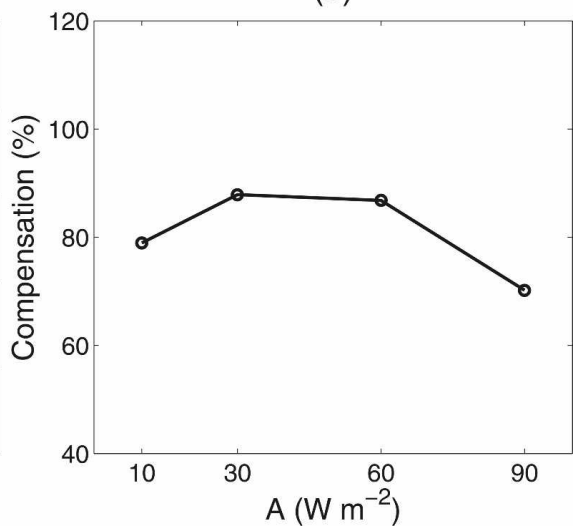

FIG. 4. (a) The location of the ITCZ (solid) and the energy flux equator (dashed), and (b) the degree of compensation $C$ by the atmospheric energy flux averaged over $20^{\circ} \mathrm{S}$ and $20^{\circ} \mathrm{N}$ as a function of the strength of forcing $A$ with control value of $\alpha(=1 X)$. 
(a)

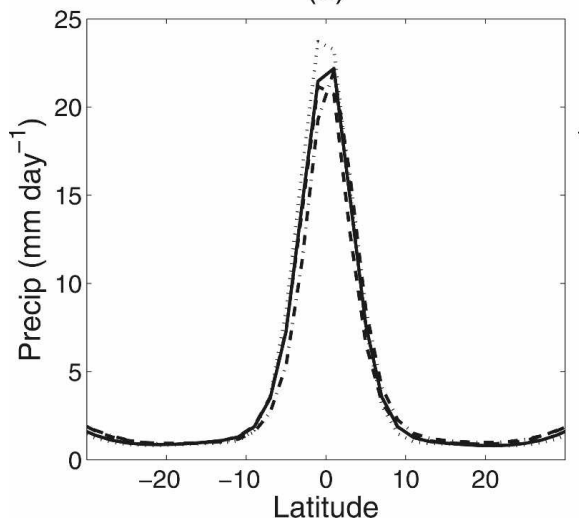

(b)

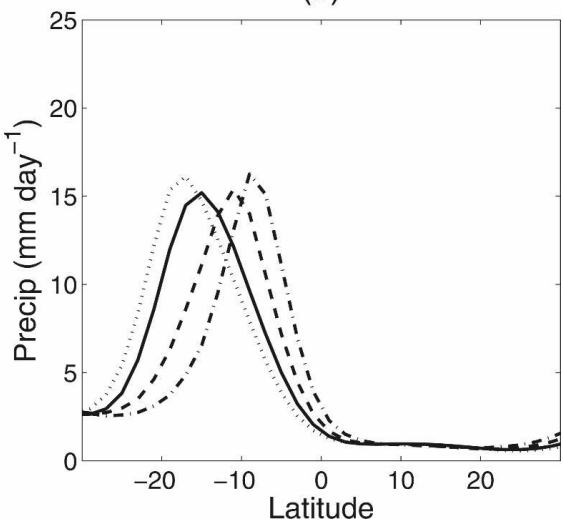

FIG. 5. Time mean, zonal mean precipitation $\left(\mathrm{mm} \mathrm{day}^{-1}\right)$ in the tropics for (a) the control runs $(A=0)$ and (b) $A=60 \mathrm{~W} \mathrm{~m}^{-2}$, with $\alpha=0+$ (dotted), $1 X$ (solid), $4 X$ (dashed), and $10 X$ (dashed-dotted).

same way, we find that the displacement of the ITCZ is generally much less than the displacement of the energy flux equator, an interesting distinction that we hope to discuss elsewhere.

The degree of flux compensation in the tropics in these experiments is shown in Fig. 4b, averaging $C$ over the region between $20^{\circ} \mathrm{S}$ and $20^{\circ} \mathrm{N}$. There is some variation with the amplitude $A$, with values ranging from $70 \%$ to $88 \%$, but the variation is not monotonic and there is some sensitivity in the precise values to the domain over which the fluxes are averaged. By computing the prediction of $\theta_{F}$, given the value of $C$ in Fig. $4 \mathrm{~b}$, as the latitude where $F_{\mathrm{ctl}}-C F_{0}=0$, we find values that are nearly identical to those in Fig. 4a, implying that a tropical average of $C$ can be converted into an estimate of the energy flux equator. In these experiments, at least, $C$ can also be converted into an estimate of the latitude of the ITCZ.

Given that $C$ is relatively close to unity, one might suspect that there is a fundamental dynamical constraint that results in near-perfect cancellation of atmospheric and oceanic fluxes in this configuration. If changing parameters in the model can change the value of $C$ substantially, this would suggest that there is no such dynamical constraint. We proceed to describe such a parameter variation.

\section{Varying the convection scheme}

Additional simulations were designed to investigate how sensitive the response of the ITCZ is to aspects of the model's moist convection scheme. We chose to focus here on one of the convective scheme parameters that modifies the extent to which convection is inhibited in the model. In AM2, this inhibition can be modi- fied by changing the parameter $\alpha$ appearing in the definition of $\lambda_{0}$ as described in the introduction and as illustrated in Held et al. (2007). Here, $A$ is set to be 60 $\mathrm{W} \mathrm{m}{ }^{-2}$, and the standard value $\alpha$ is multiplied by successive factors of $0+, 1,2,4$, and 10 . The notation $0+$ refers to a model in which only the nonentraining deep convective plume is eliminated (see Held et al. 2007 for details). With larger $\alpha$, the typical plume entrains more dry air as it ascends, making it harder for deep convection to occur, and the fraction of large-scale condensation increases. When $\alpha$ is multiplied by $0+, 1,2,4$, and 10 , the fraction of the rainfall in the tropics $\left(30^{\circ} \mathrm{S}-30^{\circ} \mathrm{N}\right)$ that is large scale in the model is $15 \%, 27 \%, 30 \%, 35 \%$, and $40 \%$, respectively. Changing $\alpha$ does not affect the time mean precipitation in the control climate very much (as shown in Fig. 5a), but it does affect the response of this climate to the imposed interhemispheric oceanic heat flux.

Figure $5 \mathrm{~b}$ shows the precipitation response, and Fig. 6 displays the locations of the ITCZ and the energy flux equator and the degree of compensation for cases with different values of $\alpha$. When the strength of this entrainment rate limiter is increased, the degree of flux compensation $C$ decreases rapidly, and the poleward displacement of both the ITCZ and $\theta_{F}$ decreases. Once again, it is demonstrable that the average tropical value of $C$ displayed in Fig. $6 \mathrm{~b}$ can be used to reproduce the values of $\theta_{F}$ in Fig. 6 a.

Some of these experiments have also been performed with a 50-m mixed layer. They are run for $30 \mathrm{yr}$ and averaged over the last $9 \mathrm{yr}$. In the control run with a deeper mixed layer, the ITCZ becomes sharper with a greater precipitation maximum (Fig. 7a, in red). SSTs also change as the mixed layer depth changes. With the control value of $\alpha$, the SST is $1.3 \mathrm{~K}$ larger at the equator 
(a)

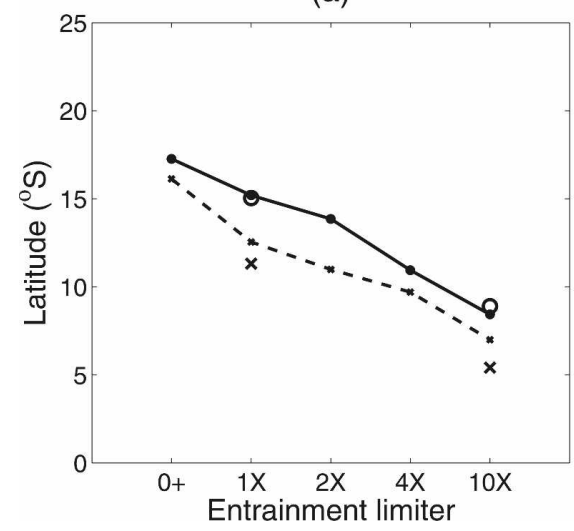

(b)

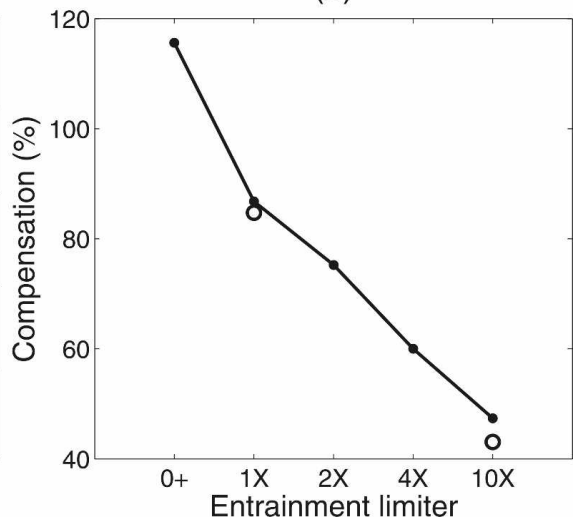

FIg. 6. Same as Fig. 4, but as a function of $\alpha$ with $A=60 \mathrm{~W} \mathrm{~m}^{-2}$. Circles (crosses) in (a) denote the position of the ITCZ (energy flux equator) for a 50-m mixed layer. Circles in (b) denote the compensation percentage for a 50-m mixed layer.

than in the 2.4-m case, and it is $2 \mathrm{~K}$ larger when $\alpha=$ $10 X$. These differences are interesting, and their explanation is not self-evident; however, the response of the precipitation to the imposed oceanic flux is very similar in this deeper mixed layer model to that in the $2.4-\mathrm{m}$ model, as illustrated for the $A=60$ case in Fig. 7b. The location of the ITCZ and the energy flux equator and the degree of compensation $C$ also do not depend significantly on the mixed layer depth, as indicated by the large black circles and crosses in Fig. 6 .

The response of the tropical circulation and precipitation to extratropical thermal forcing is clearly strongly sensitive to the convective closure in this model. Given the potential importance of this type of extratropicaltropical interaction for problems ranging from ice age simulations to the response of the tropics to variations in the Atlantic meridional overturning circulation or to
Northern Hemisphere aerosol forcing, it is important to try to understand this sensitivity and to determine the extent to which the results are model dependent. Changes in clouds underlie the sensitivity indicated in Figs. 5 and 6, which we examine in more detail in the next section.

\section{Cloud responses}

\section{a. The cloud effects on energy fluxes}

From the perspective of energy transports, the influence of extratropical thermal forcing on the tropics can be thought of as a two-step process. As a first step, cooling in the Northern Hemisphere, for example, results in increased poleward eddy energy fluxes that extract heat from the northern boundary of the tropics. Temperature gradients within the tropics are small as a (a)

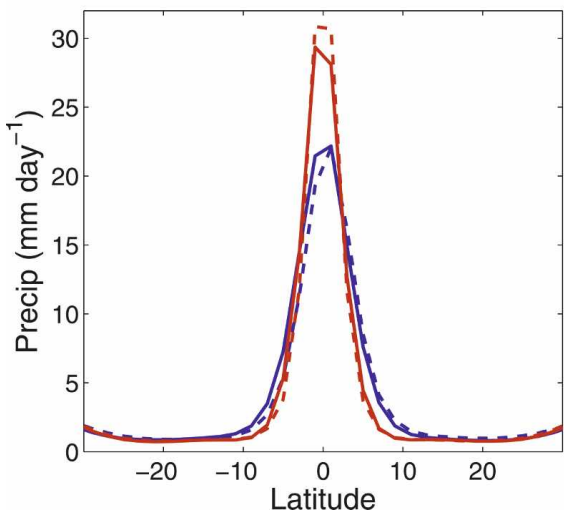

(b)

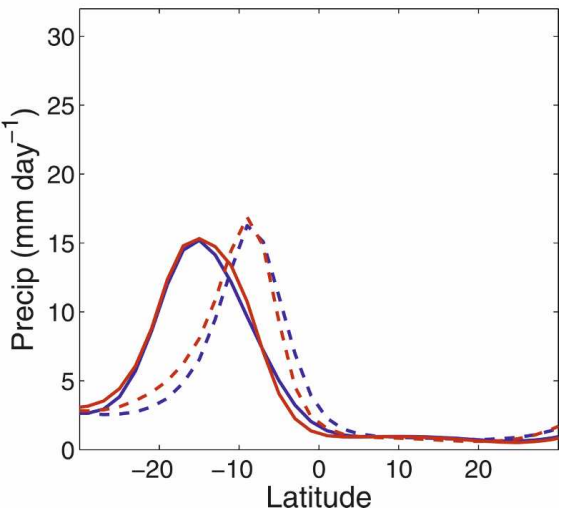

FIG. 7. Time mean, zonal mean precipitation $\left(\mathrm{mm} \mathrm{day}^{-1}\right)$ in the tropics (a) for the control runs $(A=0)$, and (b) for $A=60 \mathrm{~W} \mathrm{~m}^{-2}$. Solid (dashed) lines indicate $\alpha=1 X(10 X)$; blue (red) indicates a 2-m (50-m) mixed layer. 
consequence of the smallness of the Coriolis parameter, so the atmosphere has difficulty in balancing this cooling through local temperature changes that alter radiative fluxes. Instead, as a second step, the cooling is distributed throughout the tropics; however, as described by Lindzen and Hou (1988), one must ensure energy balance on both sides of the ITCZ (or more precisely, on both sides of the energy flux equator). Therefore, the ITCZ moves to the warmer hemisphere, making a larger proportion of the absorbed solar available to the cross-equatorial Hadley cell for distribution to the cooled hemisphere. Clouds and water vapor complicate this picture by creating gradients in the energy balance at the top of the tropical atmosphere that are not constrained to be small. An additional complication is that the original communication of the forcing to the tropics by eddies can take place through momentum fluxes as well as through energy fluxes, with asymmetric momentum fluxes contributing to the generation of interhemispheric asymmetry in the tropical flow. We do not focus here on the specifics of the eddy dynamics communicating between the extratropics and tropics; instead, we focus on the importance of the cloud responses.

Clouds can affect extratropical-tropical interactions in at least two ways. Extratropical cloud responses can alter the effective strength of the extratropical thermal forcing directly, after which the influence on the tropics proceeds as before, but with altered strength. Alternatively, in response to changes in tropical circulation, the tropical cloud cover can change to alter the energy balance and feed back on these tropical changes. For example, to the extent that enhanced subsidence is created in the tropics of the cooled hemisphere and this subsidence favors low-level clouds, the resulting cooling will enhance the tropical interhemispheric asymmetry by requiring stronger cross-equatorial fluxes (see, e.g., the discussion in Philander et al. 1996). In this section, we analyze the cloud radiative forcing (CRF) within the simulations and also describe experiments in which we manipulate the model clouds directly to help sort out these different mechanisms.

The CRF is defined as the difference between the downward net radiative flux at the top of the model atmosphere and the same flux computed for clear-sky conditions. We examine this diagnostic to estimate the role of clouds in creating the sensitivity to the convection scheme parameter presented in section 3. However, it is important to point out that a change in CRF need not come from a change in the distribution of clouds. The CRF can change even if clouds are held fixed through "cloud masking" effects (Zhang et al. 1994; Soden et al. 2004). For instance, in response to a

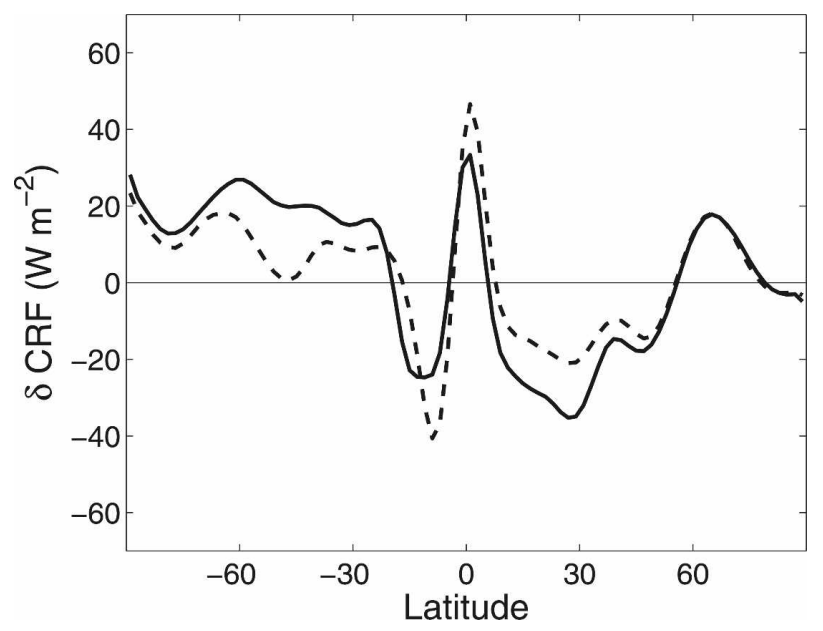

FIG. 8. The change in cloud radiative forcing for the case $A=$ $60 \mathrm{~W} \mathrm{~m}^{-2}$. Solid (dashed) lines indicate $\alpha=1 X(10 X)$.

uniform increase in water vapor content with fixed cloud distribution, the outgoing longwave radiation (OLR) is reduced more strongly in clear-sky conditions because clouds act to mask the increases in water vapor. Thus, the change in CRF in this situation will be negative, even though clearly none of this change in CRF results from changes in clouds. In this section, we will also consider simulations with fixed clouds to ensure that changes we find in CRF are indeed caused by changes in clouds rather than by cloud masking.

In Eq. (1), $R_{\mathrm{TOA}}$ can be divided into CRF and the clear-sky flux. Each of these terms can also be divided into a global mean component and a deviation from the global mean. Our interest here is in the deviation from the global mean, which we can express as a divergence of a flux. Thus, $F_{\mathrm{TOA}}$, the equivalent flux form of $R_{\mathrm{TOA}}$, can be written as $F_{\mathrm{TOA}}=F_{\mathrm{clr}}+F_{\mathrm{CRF}}$, where $F_{\mathrm{clr}}$ is the clear-sky component and $F_{\mathrm{CRF}}$ is the flux form of CRF, with $\mathrm{CRF}=-\boldsymbol{\nabla} \cdot F_{\mathrm{CRF}}$. Then the perturbed energy budget, with the global mean removed, is

$$
-\boldsymbol{\nabla} \cdot \delta F_{\mathrm{clr}}-\boldsymbol{\nabla} \cdot \delta F_{\mathrm{CRF}}-\boldsymbol{\nabla} \cdot \delta F_{0}=\boldsymbol{\nabla} \cdot \delta F,
$$

where $\delta$ denotes the difference from the control run $(A=0)$. The change in CRF $(\delta \mathrm{CRF})$ due to the extratropical forcing $[(A=60)-(A=0)]$ is plotted in Fig. 8 for two values of $\alpha$. The equivalent flux perturbation $\delta F_{\mathrm{CRF}}$ is shown in Fig. 3 (dashed-dotted line). The clear-sky component $\delta F_{\text {clr }}$ is also plotted in Fig. 3 as dotted lines. From Fig. 3 , we see that $\delta F_{\mathrm{CRF}}$ varies more with $\alpha$ than $\delta F_{\text {clr }}$; from the signs, we can infer that cloud forcing acts to amplify the extratropical thermal forcing, whereas the clear-sky response is a negative feedback. Because $\delta F_{\mathrm{CRF}}$ is larger in the smaller $\alpha$ case, this suggests that the positive feedback by clouds is larger in 
(a)

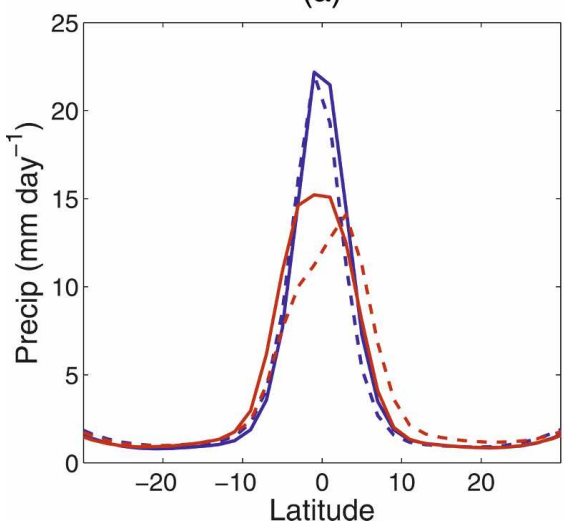

(b)

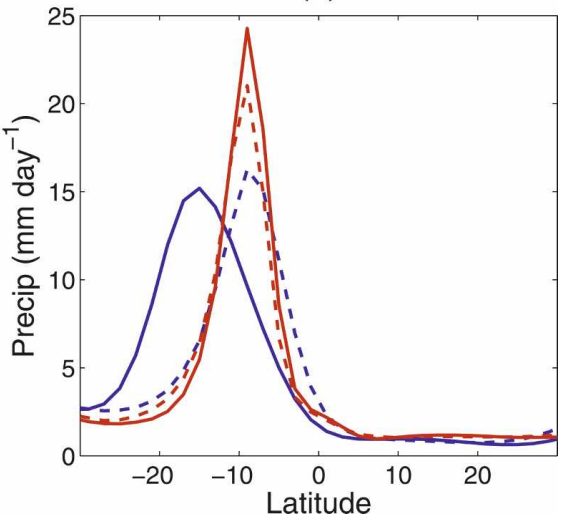

FIG. 9. Time mean zonal mean precipitation $\left(\mathrm{mm} \mathrm{day}^{-1}\right)$ in the tropics for (a) the control runs $(A=0)$ and (b) $A=60 \mathrm{~W} \mathrm{~m}^{-2}$. Solid (dashed) lines indicate $\alpha=1 X(10 X)$; blue (red) indicates the predictive (prescribed) cloud model.

the smaller $\alpha$ case, so the changes in CRF suggest that changes in clouds cause the large sensitivity to convection scheme presented in section 3 .

\section{b. The prescribed cloud simulations}

To confirm that the changes in CRF are indicative of changes in clouds rather than of cloud-masking effects, a prescribed cloud model is also examined. The prescribed cloud model allows us to examine the relative importance of cloud changes in different latitude bands. To produce a prescribed cloud distribution that also has realistic variability, we extract a 1-yr time series of the cloud water mixing ratio, cloud ice mixing ratio, and fractional cloud from each control $(A=0)$ run, sampling these variables every $3 \mathrm{~h}$. We then insert these clouds into both the control and the perturbed $(A>0)$ runs. We repeat the same year-long cloud fields during each year of the model runs. The control simulation needs to be repeated with prescribed clouds because this process has the effect of decorrelating the clouds from the model's meteorology. If the intuition developed from the CRF diagnostics is correct, when we perturb this prescribed cloud model we can expect a significantly smaller response of the energy fluxes and ITCZ, especially in cases with small $\alpha$ that have large apparent cloud feedbacks. We also expect less sensitivity of the response to $\alpha$.

Figure 9 shows the tropical precipitation distribution for both varying (blue) and prescribed (red) cloud models with two values of $\alpha$. Note that in the control run (Fig. 9a), disrupting the correlations between clouds and precipitation causes a decrease in the ITCZ precipitation to a similar extent for both values of $\alpha$. Also, for reasons that are unclear, the ITCZ in the control run with prescribed clouds and $\alpha=10 X$ favors the Northern Hemisphere (red dashed line in Fig. 9a). Figure $9 \mathrm{~b}$ shows the corresponding precipitation distributions when $A=60$. The ITCZ shift is similar for different values of $\alpha$ in fixed cloud simulations, which supports the claim that the sensitivity to $\alpha$ is due to cloud responses. Also, this shift is much less than that in the predictive cloud simulation when $\alpha=1 X$ because the positive feedback from changes in clouds is eliminated. The asymmetry in the $\alpha=10 X$ control does not seem to affect the $(A=60)-(A=0)$ response significantly.

Figure 10a shows the change in CRF ( $\delta \mathrm{CRF}$ ) that is caused by the extratropical forcing for the prescribed cloud model. These changes in CRF come mostly from the longwave component. Because the OLR changes more in clear-sky conditions, the cloud-masking effect results in warming in the warmed hemisphere and cooling in the cooled hemisphere, implying that the cloud feedbacks are, on average, somewhat less positive than indicated by the change in cloud forcing. As shown in Fig. 10a, this change in CRF is very similar for the two cases with different values of $\alpha$, confirming that the $\alpha$ dependence of the cloud forcing response to the asymmetric heating does reflect changes in clouds rather than cloud-masking effects. Figure $10 \mathrm{~b}$ shows the result of subtracting this fixed cloud change in CRF from the change in CRF in the predicted cloud models as an estimate of the CRF change due to changes in the cloud field in those simulations.

Figure 11 shows the flux responses in the prescribed cloud simulations in the same format as Fig. 3. Prescribing clouds results in a large reduction of compensation in the $\alpha=1 X$ case: $\delta F_{\mathrm{CRF}}$ decreases in this case to the extent that it becomes similar to that in $\alpha=10 X$ case, 
(a)

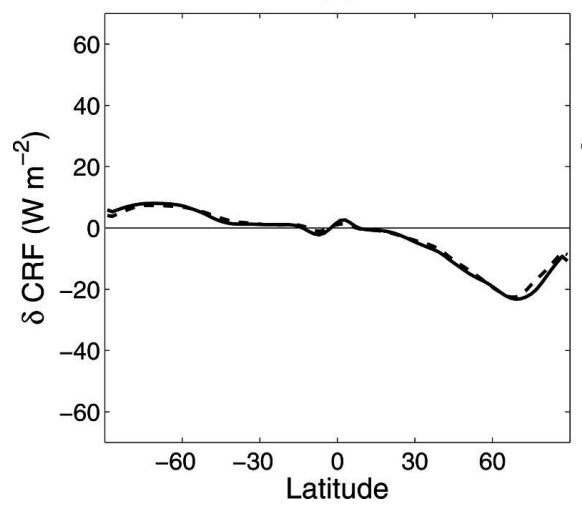

(b)

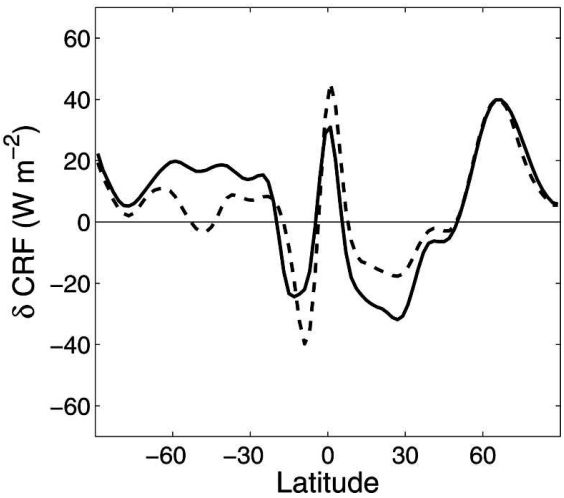

FIG. 10. The change in cloud radiative forcing (a) for the prescribed cloud model and (b) when cloud-masking effect plotted in Fig. 10a is subtracted from Fig. 8 for each value of $\alpha$. Lines as in Fig. 8.

and the change in the total atmospheric flux response $\delta F$ is similar in the two cases as well. The changes in ITCZ position and energy flux equator for prescribed and interactive clouds are summarized in Fig. 12. We conclude that altering the entrainment rate limiter in the convection scheme mainly affects the response of clouds, and this adjustment results in the very different sensitivities to the same extratropical thermal forcing.

\section{c. The sensitivity of CRF to the entrainment rate limiter}

We now turn our attention back to the predictive cloud simulations to understand how the convection parameter $\alpha$ creates this large sensitivity. The change in cloud forcing shown in Fig. 8 is primarily determined by its shortwave component (Fig. 13a), although there are longwave CRF responses that partially offset the shortwave component in the tropics. The shortwave CRF depends strongly on lower tropospheric clouds. Figure $13 \mathrm{~b}$ shows the change in the amount of low and mid(>400 mb) clouds (for brevity, we will call it low cloud) as calculated using the GCM's random overlap assumption. The sign of the low-cloud response and its sensitivity to $\alpha$ are consistent with the cloud forcing changes.

The tropical changes in cloud forcing are directly associated with the shift in the ITCZ. Note that these tropical changes counteract the imposed extratropical thermal forcing because the shortwave forcing tends to be larger than the longwave forcing in the model's regions of deep convection. Thus, if the imposed forcing were confined to the tropics, it might be expected that changes in CRF would create a negative feedback, opposite to the cases we show here. Cloud responses in (a)

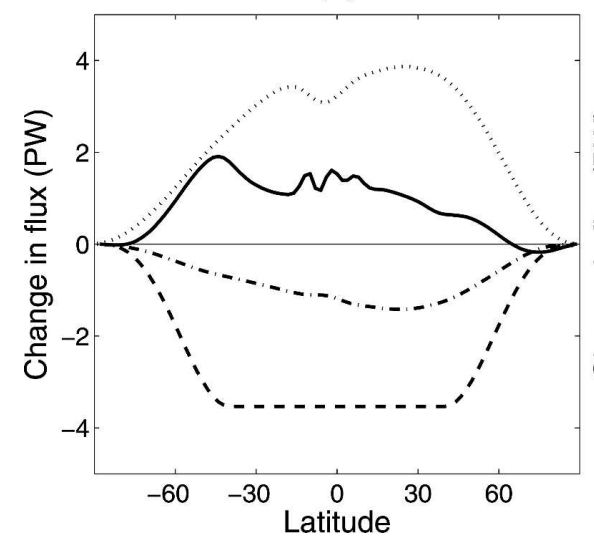

(b)

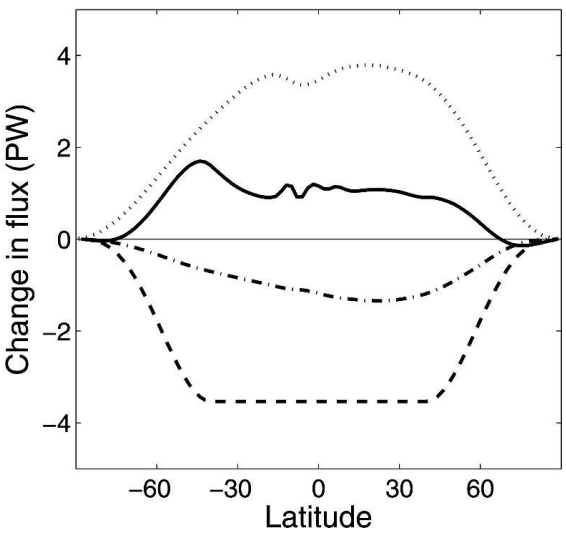

FIG. 11. Same as Fig. 3, but for the prescribed cloud models with (a) $\alpha=1 X$ and (b) $\alpha=$ $10 X$. Note that the dashed-dotted line corresponds to the equivalent flux form of Fig. 10a. 
(a)

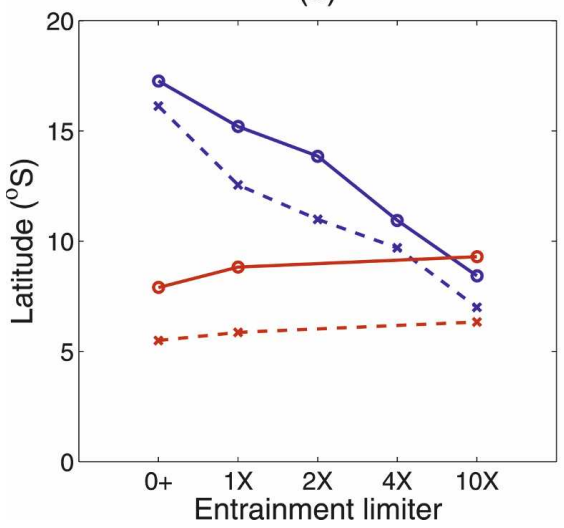

(b)

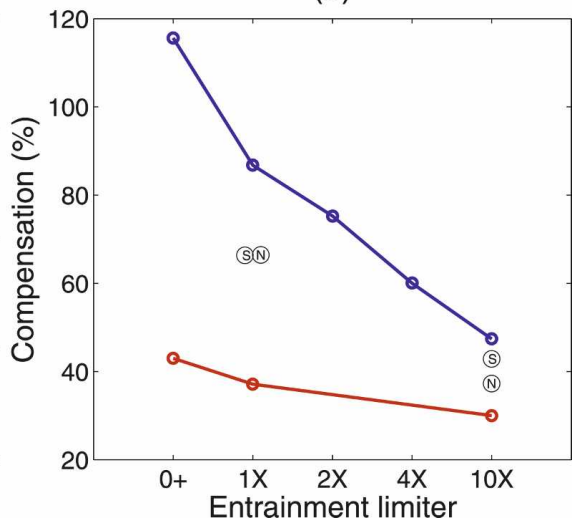

FIG. 12. Same as Fig. 6. Blue (red) indicates predictive (prescribed) cloud models. Circles in (b) are the values of compensation when clouds only in the southern or northern extratropics are fixed, denoted "S" and "N," respectively.

the northern subtropics and southern mid-to-high latitudes are responsible for strengthening the imposed asymmetric extratropical thermal forcing, as seen in the shortwave forcing plot (Fig. 13a). There is also substantial negative feedback from clouds in high latitudes in the cold Northern Hemisphere, but this response is not sensitive to the convection scheme because there is little convection in that area.

To determine which area is more important in amplifying the response of atmospheric fluxes, the southern midlatitudes $\left(30^{\circ}-90^{\circ} \mathrm{S}\right)$ or the northern subtropics $\left(10^{\circ}-30^{\circ} \mathrm{N}\right)$, experiments have been performed in which

(a)

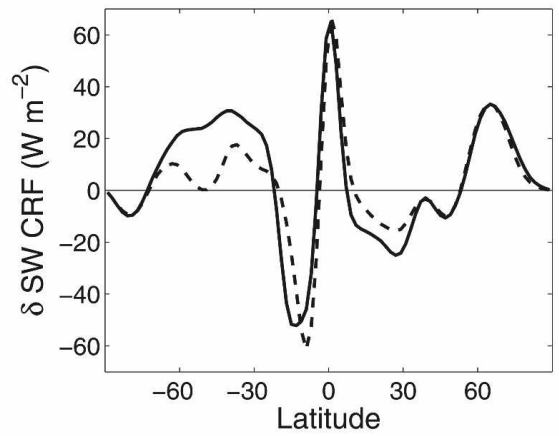

(b)

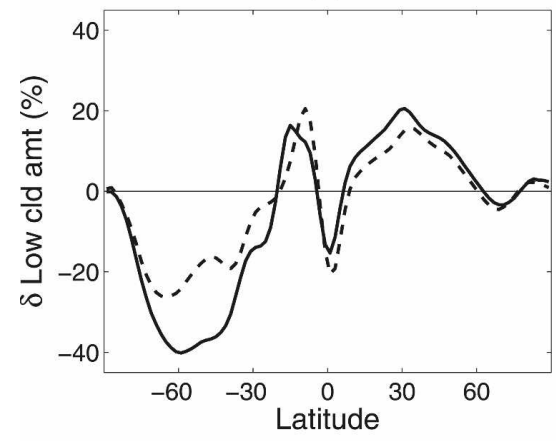

(c)

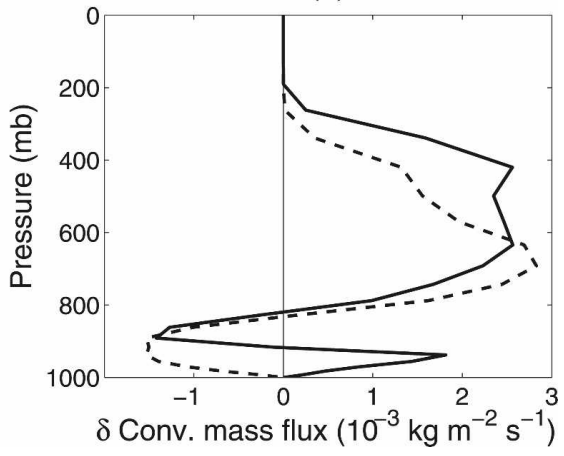

FIG. 13. (a) The change in shortwave component of cloud forcing in $\mathrm{W} \mathrm{m}^{-2}$. (b) The change in low-cloud amount (\%). (c) The change in convective mass flux averaged over $90^{\circ}$ and $30^{\circ} \mathrm{S}$ in $10^{-3} \mathrm{~kg} \mathrm{~m}^{-2} \mathrm{~s}^{-1}$. All plots are for the case $A=60 \mathrm{~W} \mathrm{~m}^{-2}$. Lines as in Fig. 8. 
clouds have been prescribed in only one of these areas in turn. These regions were chosen as those in which the shortwave cloud forcing and low-cloud responses in Figs. 13a and 13b are most sensitive to $\alpha$. We find that each of these regions contributes roughly half of the cloud feedback effect on the degree of tropical compensation $C$, as illustrated in Fig. 12b.

Both the reduction in low-cloud amount over the southern midlatitudes and the increase in low-cloud amount over the northern subtropics are sensitive to $\alpha$. In the warmed Southern Hemisphere, the temperature and humidity near the surface increase so that the atmosphere is destabilized, thereby inducing more deep convection, as can be seen from Fig. 13c, which shows the change in convective mass flux averaged over $90^{\circ}-$ $30^{\circ} \mathrm{S}$. Because deep convective mass flux increases at the expense of shallow convection and drives the compensating subsidence, there is a reduction in convective mass flux below $800 \mathrm{mb}$. In case of $\alpha=1 X$, shallow convection seems to become shallower as the convective mass flux increases below $900 \mathrm{mb}$. Hence, increasing deep convection directly contributes to the reduction in lower tropospheric cloud amount because deep convection warms and dries the lower troposphere through the compensating subsidence. This destroys lower-level clouds and/or prevents their formation. These aspects of convection-cloud interactions are likely to be model dependent. Deep convection occurs more frequently when $\alpha$ is smaller. Thus, in the southern midlatitudes, the smaller the value of $\alpha$, the larger the increase of deep convection and the larger the reduction of low-cloud amount, which in turn leads to more warming through the feedback of cloud forcing on TOA energy fluxes. The opposite changes occur in the northern subtropics.

In the cooled Northern Hemisphere, the sensitivity of the cloud response (Fig. 13b) to deep convection occurs in lower latitudes than in the warmed Southern Hemisphere. Although somewhat weaker than the changes in the Southern Hemisphere, they are evidently of comparable importance for the tropical responses because of greater proximity to the equator. The sensitivity to convection is presumably confined to lower latitudes in the cooled hemisphere because there is very little deep convection in the control case at higher latitudes, and imposed cooling cannot further decrease deep convection.

In addition to the response to local temperature change, enhanced subsidence over the northern subtropics associated with a stronger Hadley circulation in the cooled hemisphere can induce an increase in lowcloud amount. Thus, it is possible that the sensitivity of the cloud feedbacks to convection in the northern sub- tropics is a consequence of changes in convection in the warm Southern Hemisphere, which increase the asymmetry of the Hadley circulation and thereby induce cloud changes in the northern subtropics through increased subsidence. For any given $\alpha$, it is difficult to determine whether strengthening of subsidence or reduction in deep convection due to a stabilized atmosphere is more important in increasing the low-cloud amount in the northern subtropics. However, our prescribed cloud simulations can help sort out which effect is more responsible for creating the sensitivity to $\alpha$. When we prescribe clouds only in the southern extratropics, removing that source of feedback, the sensitivity of cloud response to $\alpha$ in the northern subtropics is only reduced by $30 \%$. Therefore, we conclude that over the northern subtropics, the sensitivity of $\delta \mathrm{CRF}$ to $\alpha$ shown in Fig. 8 can be attributed to the sensitivity of deep convection to $\alpha$ in response to local temperature changes rather than to strengthened subsidence in the descending branch of the Hadley cell.

The dependence of the precipitation response on cloud feedbacks suggests an important way in which uncertainties in cloud modeling can create uncertainties in regional responses to climatic perturbations.

\section{Conclusions}

In this study, the response of the ITCZ to extratropical heating and cooling is investigated. An aquaplanet GCM coupled to a slab ocean is perturbed by an imposed cross-equatorial oceanic flux. The ITCZ is displaced toward the warmer hemisphere. In thinking about the ITCZ displacement, we find it convenient to focus also on the degree of compensation of the imposed oceanic transport by the atmospheric energy transport in the tropics. We can relate this degree of compensation to the latitude of the energy flux equator where the atmospheric energy transport vanishes, which provides insight into the position of the ITCZ.

This degree of compensation is relatively high (greater than $70 \%$ ) in the standard configuration of the model, and it might be tempting to conclude that a high degree of compensation is somehow assured by the nature of large-scale atmospheric dynamics. However, the convection scheme in the model can be altered to vary the degree of compensation from $47 \%$ to $115 \%$ for otherwise identical models and forcing. Simulations with prescribed clouds have been used to show that changes in the cloud response to the differential heating of the two hemispheres are primarily responsible for this sensitivity to the convection scheme.

It is commonly observed that cloud feedbacks are a source of much of the uncertainty in estimates of global 
mean climate responses to external forcing (Cess et al. 1996). This study provides evidence that important aspects of the regional response of climate to external forcing are also sensitive to cloud feedbacks. The model configuration utilized here can be considered as a benchmark computation that can be used to compare this important aspect of tropical-extratropical feedback in a wide range of GCMs. This type of model comparison study may be very useful in helping to understand intermodel differences in the response of the tropics to ice sheets, variations in meridional overturning, or aerosol forcing confined to one hemisphere.

Acknowledgments. We thank Rong Zhang, Ken Takahashi, and three anonymous reviewers for very helpful comments on earlier versions of this paper. Special thanks to Rong Zhang for helping with the prescribed cloud simulations. This work was supported by NSF Grant ATM-0612551.

\section{REFERENCES}

Anderson, J. L., and Coauthors, 2004: The new GFDL global atmosphere and land model AM2-LM2: Evaluation with prescribed SST simulations. J. Climate, 17, 4641-4673.

Broccoli, A. J., K. A. Dahl, and R. J. Stouffer, 2006: Response of the ITCZ to Northern Hemisphere cooling. Geophys. Res. Lett., 33, L01702, doi:10.1029/2005GL024546.

Cess, R. D., and Coauthors, 1996: Cloud feedback in atmospheric general circulation models: An update. J. Geophys. Res., 101 (D8), 12 791-12 794.

Chiang, J. C. H., and C. M. Bitz, 2005: Influence of high-latitude ice cover on the marine intertropical convergence zone. Climate Dyn., 25, 477-496.

Frierson, D. M. W., I. M. Held, and P. Zurita-Gotor, 2006: A gray-radiation aquaplanet moist GCM. Part I: Static stability and eddy scale. J. Atmos. Sci., 63, 2548-2566.

Held, I. M., M. Zhao, and B. Wyman, 2007: Dynamic radiative- convective equilibria using GCM column physics. J. Atmos. Sci., 64, 228-238.

Koutavas, A., and J. Lynch-Stieglitz, 2004: Variability of the marine ITCZ over the eastern Pacific during the past 30,000 years: Regional perspective and global context. The Hadley Circulation: Present, Past, and Future, H. F. Diaz and R. S. Bradley, Eds., Springer, 347-369.

Lea, D. W., D. K. Pak, L. C. Peterson, and K. A. Hughen, 2003: Synchroneity of tropical and high-latitude Atlantic temperatures over the last glacial termination. Science, 301, 13611364.

Lee, M.-I., M. J. Suarez, I.-S. Kang, I. M. Held, and D. Kim, 2008: A moist benchmark calculation for the atmospheric general circulation models. J. Climate, in press.

Lindzen, R. S., and A. Y. Hou, 1988: Hadley circulations for zonally averaged heating centered off the equator. J. Atmos. Sci., 45, 2416-2427.

Moorthi, S., and M. J. Suarez, 1992: Relaxed Arakawa-Schubert: A parameterization of moist convection for general circulation models. Mon. Wea. Rev., 120, 978-1002.

Neale, R. B., and B. J. Hoskins, 2000: A standard test for AGCMs including their physical parameterizations. I: The proposal. Atmos. Sci. Lett., 1, 101-107.

Philander, S. G. H., D. Gu, D. Halpern, G. Lambert, N.-C. Lau, T. Li, and R. C. Pacanowski, 1996: Why the ITCZ is mostly north of the equator. J. Climate, 9, 2958-2972.

Soden, B. J., A. J. Broccoli, and R. S. Hemler, 2004: On the use of cloud forcing to estimate cloud feedback. J. Climate, 17, 3661-3665.

Tokioka, T., K. Yamazaki, A. Kitoh, and T. Ose, 1988: The equatorial 30-60-day oscillation and the Arakawa-Schubert penetrative cumulus parameterization. J. Meteor. Soc. Japan, 66, 883-901.

Xie, S.-P., 2004: The shape of continents, air-sea interaction, and the rising branch of the Hadley circulation. The Hadley Circulation: Present, Past, and Future, H. F. Diaz and R. S. Bradley, Eds., Springer, 121-152.

Zhang, M. H., J. J. Hack, J. T. Kiehl, and R. D. Cess, 1994: Diagnostic study of climate feedback processes in atmospheric general circulation models. J. Geophys. Res., 99, 5525-5537.

Zhang, R., and T. L. Delworth, 2005: Simulated tropical response to a substantial weakening of the Atlantic thermohaline circulation. J. Climate, 18, 1853-1860. 